\title{
Colangiopancreatografía retrógrada endoscópica en pacientes con asa en Y-de-Roux*
}

\author{
Drs. MARCELO FALCÃO ${ }^{1}$, JOSEMBERG M. CAMPOS ${ }^{1}$, MANOEL GALVÃO NETO ${ }^{1}$, ALMINO RAMOS ${ }^{1}$, \\ EDUARDO FRANCA ${ }^{1}$, ÁLVARO FERRAZ ${ }^{1}$, CÉSAR MUÑOZ C. ${ }^{2}$, ALEX ESCALONA P. ${ }^{2}$
}

1 Hospital da Clínicas, Departamento de Cirugia - Universidad Federal de Pernambuco, Recife, Brasil.

2 Departamento de Cirugía Digestiva, Pontificia Universidad Católica de Chile.

Chile.

\begin{abstract}
Endoscopic retrograde cholangiopancreatography in Roux-en-Y patients

The reconstruction of the digestive tract with a Roux-en-Y anastomosis has been performed more frequently in the last years mainly due to the overgrowth in the surgical treatment of obesity by gastric bypass in Roux-en-Y. The obesity pandemic is also associated with an increase in both the preoperative and postoperative diagnosis of gallstones in this population. Bearing in mind the greater complexity of endoscopic retrograde cholangiopancreatography in these circumstances, the authors have reviewed the various endoscopic approaches available, evaluating 249 articles published between 1990 and 2010 obtained from Medline, Cochrane and Scielo, excluding case reports and articles outside the specific scope of this study. Endoscopic techniques with high success rates include the use of enteroscope, colonoscope, enteral and transgastric access combined with surgery. Even with the technological progress already made, further development of the instruments used will be necessary if greater comfort and safety is to be achieved with this procedure.
\end{abstract}

Key words: Choledocholithiasis (MeSH), Anastomosis, Roux-en-Y (MeSH), Gastric Bypass (MeSH), Cholangiopancreatography, Endoscopic Retrograde (MeSH).

\section{Resumen}

La reconstrucción del trato digestivo con un asa en Y-de-Roux ha sido más empleada en los últimos años debido principalmente al aumento del tratamiento quirúrgico de la obesidad mediante el bypass gástrico en Y-de-Roux (BGYR). La pandemia de la obesidad se ha asociado a un aumento en la prevalencia de litiasis biliar en esta población, diagnosticada en el estudio preoperatorio y durante el seguimiento postoperatorio. La colangiopancreatografía endoscópica retrógrada (CPRE) posee una mayor complejidad en estas circunstancias donde existe una alteración anatómica que dificulta el acceso a la papila mayor. El objetivo de esta revisión es describir las alternativas de acceso al asa biliopancreática para realizar CPRE en pacientes con un asa en Y-deRoux enfatizando la accesibilidad técnica, los resultados y complicaciones de los procedimientos. Se realizó una búsqueda y revisión de la literatura en las bases de datos: Medline, Cochrane y Scielo. Se evaluaron 249 publicaciones desde 1990 a 2010, en idioma español, inglés y portugués. Fueron excluidos de esta revisión los reportes de casos y publicaciones no atingentes. Las opciones endoscópicas para realizar CPRE en pacientes

*Recibido el 2 de enero de 2012 y aceptado para publicación el 12 de febrero de 2012.

Correspondencia: Dr. Josemberg M. Campos

Rua Vigário Barreto, 127 / 802 - Graças, 52020-140, Recife, Brasil.

berg@elogica.com.br 
con reconstrucción con un asa en Y-de-Roux incluyen: el uso de enteroscopio, colonoscopio, acceso enteral y transgástrico combinado con cirugía. Todos las alternativas presentan una alta tasa de éxito, sin embargo, pese a los avances tecnológicos obtenidos en los últimos años en el instrumental utilizado aún se necesitan herramientas que permitan una mayor comodidad y seguridad para este procedimiento.

Palabras clave: Coledocolitiasis, anastomosis, Y de Roux, bypass gástrico, colangiopancreatografía endoscópica retrógada.

\section{Introducción}

La anastomosis gastrointestinal en Y-de-Roux es una técnica de reconstrucción del tránsito intestinal creada para desviar la secreción biliopancreática e impedir su reflujo al estómago ${ }^{1,2}$. La pandemia de la obesidad posicionó al bypass gástrico en Y-deRoux (BGYR) como uno de los procedimientos quirúrgicos más empleados para tratar esta enfermedad $^{3}$. Entre las complicaciones a largo plazo del BGYR destaca las litiasis de la vesícula biliar y la coledocolitiasis, condiciones que estarían asociadas a cambios en la composición de la bilis, secundario a la baja de peso y la alteración del circuito enterohepático que provocaría el asa en Y-de-Roux ${ }^{4,5}$.

La colangiopancreatografía retrógrada endoscópica (CPRE) fue descrita en 1968 y la introducción de la papilotomía en 1974. En pacientes con anatomía preservada, el colangiograma obtenido por la inyección endoscópica de medio de contraste a través del orificio papilar, seguido por la sección diatérmica del esfínter de Oddi y barrido del conducto biliar presentan un $95 \%$ de sensibilidad y $100 \%$ de especificidad para el diagnóstico de la coledocolitiasis ${ }^{6}$. El éxito técnico de este procedimiento es mayor al $90 \%$, con una morbididad de $5 \%$ y mortalidad menor al $1 \%$ en centros de referencia ${ }^{7,8}$.

El BGYR implica la exclusión duodenal y de la papila mayor del tránsito normal del tubo digestivo, prácticamente impidiendo el acceso y por tanto, el tratamiento endoscópico de la litiasis de la vía biliar principal empleando equipamientos convencionales debido a la mayor distancia y tortuosidad de las asas yeyunales ${ }^{1,9,10}$. En esta situación, se ha sugerido el acceso a la papila duodenal mayor a través de un enteroscopio, colonoscopio o duodenoscopio convencional introducido a través de una ostomía ${ }^{11}$.

El objetivo de esta revisión es describir las alternativas de acceso al asa biliopancreática para realizar CPRE en pacientes con un asa en Y-de-Roux enfatizando la accesibilidad técnica, los resultados y complicaciones de los procedimientos.

\section{Metodología de búsqueda y selección de la literatura}

Se realizó una búsqueda de la literatura en las bases de datos: Medline, Cochrane y Scielo. Los términos de búsqueda utilizados fueron: endoscopic surgery AND bariatric surgery, Roux-en-Y gastric bypass AND bariatric surgery, choledocolithiasis AND Roux-en-Y gastric bypass, ERCP AND bariatric surgery, enteroscopy AND ERCP; y transgastric ERCP AND bariatric surgery. Como resultado de la estrategia de búsqueda utilizada se obtuvo 249 artículos publicados entre los años 1990 y 2010 en los idiomas inglés, español y portugués. Posterior a la lectura del resumen se excluyeron los reportes de casos y las revisiones narrativas. Finalmente, se consideraron atingentes para esta revisión 17 estudios, cuyos resultados son presentados en el texto que sigue a continuación (Tabla 1).

\section{Resultados}

\section{CPRE convencional}

La descripción original de la reconstrucción en Y-de-Roux consta de un asa eferente de aproximadamente $50 \mathrm{~cm}$ de longitud y un asa alimentaria o aferente de 20-30 cm. La anatomía de esta reconstrucción permite la realización de una CPRE convencional en cerca de 25\% de los casos (Figura $1)^{12}$. Junto con lo anterior, en el BGYR hay un alargamiento de las asas aferente y eferente del reconstrucción en Y-de-Roux lo que provoca una mayor dificultad en la progresión y evaluación endoscópica convencional por vía oral ${ }^{1}$.

En 1997, Hintze et $\mathrm{al}^{13}$, describieron una tasa 33\% de identificación de la papila con duodenoscopio convencional en casos de asa eferente corta. En 2002, Wright et $\mathrm{al}^{14}$, describieron la realización de CPRE en derivación gástrica usando inicialmente un endoscopio de visión frontal. El hilo guía era posicionado preferentemente en el estómago excluso con posterior paso del duodenoscopio sobre el hilo-guía bajo control fluoroscopio. La CPRE fue intentada en 15 pacientes, alcanzando la papila en el $67 \%$ de los casos y el conducto biliar canulado en la totalidad de ellos. Se presentaron 3 (12\%) complicaciones en 25 procedimientos realizados, incluyendo dos casos de pancreatitis y un sangrado leve, todos ellos tratados médicamente. La imposibilidad de ejecución del procedimiento en todos los casos confirma la dificultad de esta técnica a través de la vía oral, incluso en centros con amplia experiencia (Figura 2). 
Tabla 1. Cuadro de referencias utilizadas en esta revisión

\begin{tabular}{|c|c|c|c|c|c|c|c|}
\hline Autor / año & Estudio & Pacientes & Equipamiento & $\begin{array}{l}\text { Acceso } \\
\text { papilar }\end{array}$ & CPRE & $\begin{array}{l}\text { Compli- } \\
\text { caciones }\end{array}$ & Acceso biliar \\
\hline Hintze / 1997 & Serie casos & 65 & Duodenoscopio & $33 \%$ & $100 \%$ & & Oral \\
\hline Wright / 2002 & Serie casos & 15 & $\begin{array}{l}\text { Colono ped. } \\
\text { duodenoscopio }\end{array}$ & $\begin{array}{l}13 \% \\
53 \%\end{array}$ & $\begin{array}{c}0 \% \\
100 \%\end{array}$ & $12 \%$ & $\begin{array}{l}\text { Oral } \\
\text { Oral }\end{array}$ \\
\hline Elton / 1998 & Serie casos & 18 & $\begin{array}{l}\text { Enteroscopio colono } \\
\text { ped. }\end{array}$ & $\begin{array}{l}86 \% \\
82 \%\end{array}$ & $94 \%$ & & Oral \\
\hline Neumann / 2009 & Serie casos & 13 & $\begin{array}{l}\text { Enteroscopio de balón } \\
\text { único }\end{array}$ & $61 \%$ & $46 \%$ & & Oral \\
\hline Wang AY / 2010 & Serie casos & 13 & $\begin{array}{l}\text { Enteroscopio de balón } \\
\text { único }\end{array}$ & $92,3 \%$ & $90 \%$ & cero & Oral \\
\hline Parlak / 2010 & Serie casos & 14 & $\begin{array}{l}\text { Enteroscopio doble } \\
\text { balón }\end{array}$ & $92,9 \%$ & $100 \%$ & $7,1 \%$ & Oral \\
\hline Emmett / 2007 & Serie casos & 14 & $\begin{array}{l}\text { Enteroscopio doble } \\
\text { balón }\end{array}$ & $85 \%$ & $80 \%$ & & Oral \\
\hline Aabakken 2007 & Serie casos & 13 & $\begin{array}{l}\text { Enteroscopio doble } \\
\text { balón }\end{array}$ & $100 \%$ & $100 \%$ & cero & Oral \\
\hline Kuga / 2008 & Serie casos & 6 & $\begin{array}{l}\text { Enteroscopio doble } \\
\text { balón }\end{array}$ & $83,3 \%$ & $83,3 \%$ & cero & Oral \\
\hline Chu YC / 2008 & Serie casos & 5 & $\begin{array}{l}\text { Enteroscopio doble } \\
\text { balón }\end{array}$ & $60 \%$ & $60 \%$ & & Oral \\
\hline Monkemuller / 2009 & Prospectivo & 11 & $\begin{array}{l}\text { Enteroscopio doble } \\
\text { balón }\end{array}$ & $82 \%$ & $58 \%$ & $5,80 \%$ & Oral \\
\hline Matlock / 2005 & Serie casos & 14 & Duodenoscopio & $100 \%$ & $100 \%$ & & Gastrostomía \\
\hline Martínez / 2006 & Serie casos & 6 & $\begin{array}{l}\text { Duodenoscopio } \\
\text { terapéutico }\end{array}$ & $50 \%$ & $67 \%$ & & $\begin{array}{l}\text { Gastrostomía } \\
\text { percutánea }\end{array}$ \\
\hline Серpa / 2007 & Serie casos & 10 & Duodenoscopio & $50 \%$ & $80 \%$ & & $\begin{array}{l}\text { Transgástrica } \\
\text { laparoscópica }\end{array}$ \\
\hline Lopes / 2009 & Serie casos & 9 & Duodenoscopio & $90 \%$ & $100 \%$ & $33 \%$ & $\begin{array}{l}\text { Transgástrica } \\
\text { laparoscópica }\end{array}$ \\
\hline Patel / 2008 & Serie casos & 8 & Duodenoscopio & $100 \%$ & $100 \%$ & & Transgástrica \\
\hline Salleem / 2010 & Serie casos & 50 & $\begin{array}{l}\text { Enteroscopio de balón } \\
\text { único }\end{array}$ & $70 \%$ & $91 \%$ & & Oral \\
\hline
\end{tabular}

\section{CPRE con el empleo del colonoscopio pediátrico}

Este método fue inicialmente relatado en 1988 por Gostout et al ${ }^{15}$. En 1998 Elton et al ${ }^{16}$, presentaron 18 pacientes con reconstrucción en Y-de-Roux sometidos a 25 intentos de CPRE usando un enteroscopio. La identificación de la papila o entero-entero anastomosis fue posible en el $86 \%$ de los pacientes, mientras que con el colonoscopio pediátrico se obtuvo un $82 \%$ de éxito. La canulación de la vía biliar fue exitosa en la mayoría de los casos con papila nativa (4/5 pacientes). En 2/4 pacientes (50\%) se logró realizar canulación pancreática. La literatura registra pocos casos de CPRE usando colonoscopio pediátrico, lo que dificulta una evaluación más crítica.

\section{CPRE con el empleo de enteroscopio de balón único}

El uso de este procedimiento ha sido ampliado debido a la ventaja de disminuir la formación de un loop en el intestino delgado, principalmente en el acceso al asa biliopancreática de la Y-de-Roux. Neumann et $\mathrm{al}^{17}$, en una serie de 13 casos, presentan una tasa de éxito del 61\% en acceso al asa biliopancreática y 46\% en la realización de la CPRE con enteroscopio de balón único (Figura 3).

Wang et $\mathrm{al}^{18}$, comunican una serie de casos en que la enteroscopia de balón único permitió efectivamente el diagnóstico y CPRE terapéutica en pacientes con anatomía alterada por operaciones de Whipple, hepático-yeyuno anastomosis, Billroth 
II y anastomosis en Y-de-Roux. Los autores obtuvieron resultados de éxito diagnóstico en 12/13 (92,3\%) pacientes y en 13/16 (81,3\%) procedimientos. La terapéutica fue necesaria en 10 pacientes, pero tuvo éxito en nueve (90\%), habiendo dos casos de pancreatitis aguda de resolución clínica satisfactoria después de la colangiografía. No hubo mortalidad, sangrado o perforación.

Salleem et $\mathrm{al}^{19}$, describieron los resultados de 50 pacientes sometidos a 56 sesiones de CPRE por enteroscopia con balón único en enfermedad biliopancreática benigna, obteniendo éxito en el diagnóstico de 70\% (39/56) y terapéutico en 91\% (21/39) de ellos. Además en esta publicación, entre las enteroscopias de balón único con suceso, inicialmente se utilizó el colonoscopio pediátrico en 22 pacientes, sin éxito. En ellos, con el empleo del enteroscopio de balón único, fue posible la CPRE en 68\% (15/22) de los casos.

Este tipo de enteroscopia es una técnica reciente que permite el diagnóstico y la terapéutica avanzada en todo el intestino delgado ${ }^{20}$. Esta técnica presenta un riesgo aumentado de pancreatitis y necesita aún de la mejoría de los accesorios para la instrumentación biliar.

\section{CPRE con el empleo del enteroscopio de doble balón}

En los centros con experiencia en enteroscopia de doble balón se describe una tasa superior al 90\% en la identificación del asa biliopancreática y superior al 80\% en la visualización de la papila duodenal mayor en los pacientes con un asa en Y-de-Roux (Figura 4).

Parlak et $\mathrm{al}^{21}$, evaluaron 14 pacientes, presentando una tasa de éxito de 92,9\% en el acceso al asa biliar, obtuvo $100 \%$ de éxito en la cateterización de la vía biliar, presentando como principal complicación neumoperitoneo en un caso resuelto clínicamente. Emmett et $\mathrm{al}^{10}$, en una serie de 14 pacientes, obtuvieron éxito en 20 sesiones de CPRE usando enteroscopio de doble balón, logrando una identificación de la papila en $85 \%$ y cateterización de la vía biliar en $80 \%$. Aabakken et $\mathrm{al}^{22}$, el 2007 publicaron una serie con 18 CPRE en 13 pacientes, obteniendo éxito en la identificación de la vía biliar y terapéutica en $100 \%$. Kuga et $\mathrm{al}^{23}$, en una serie con seis pacientes, presentaron tasa de éxito de 83,3\%. Chu et $\mathrm{al}^{9}$, obtuvieron éxito en la cateterización biliar en 3/5 pacientes. Mönkemüller et $\mathrm{al}^{24}$, evaluaron el empleo del enteroscopio de doble balón en pacientes con anastomosis en Y-de-Roux. Los resultados obtenidos en este estudio reportan una tasa de éxito diagnóstico en $82 \%$ y terapéutico

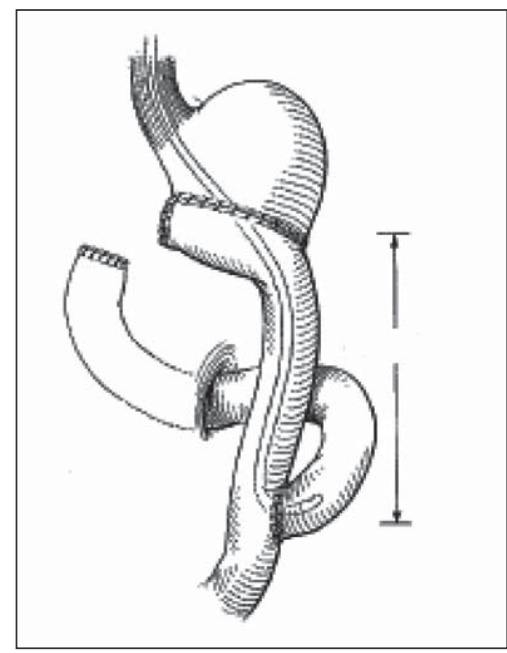

Figura 1. Acceso por vía oral del asa biliar en la cirugía de Billroth II.

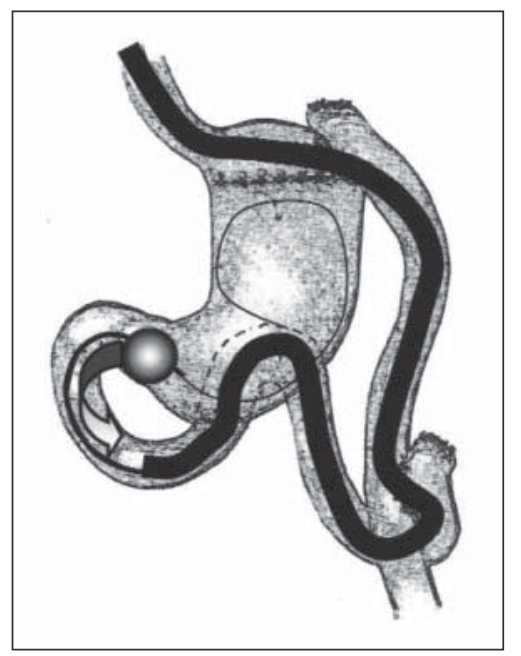

Figura 2. Wright y cols, realizaron el acceso por vía oral con duodenoscopio convencional sobre hilo guía en derivaciones gástricas.

en 58\%. Los autores relataron dificultad en la cateterización de la vía biliar debido al posicionamiento de la papila duodenal y a los accesorios disponibles para esta situación. La principal complicación fue la perforación de la anastomosis hepático-yeyunal en un paciente con coledocolitiasis recurrente que tuvo una evolución satisfactoria después del tratamiento quirúrgico.

Existen desventajas importantes en la realización de la CPRE con el enteroscopio de doble balón, tales como la falta del elevador y el canal de trabajo fino, dificultando el abordaje diagnóstico y terapéutico de la vía biliopancreática. Otra limitación de esta técnica es la falta del accesorio especialmente destinado al enteroscopio. Existe además la posibilidad de complicaciones debido a las alteraciones complejas de la anatomía, presencia de adherencias y formación de asas en el trayecto. Todas estas situaciones son menos frecuentes cuando se progresa en la curva de aprendizaje. Asimismo, las publi- 

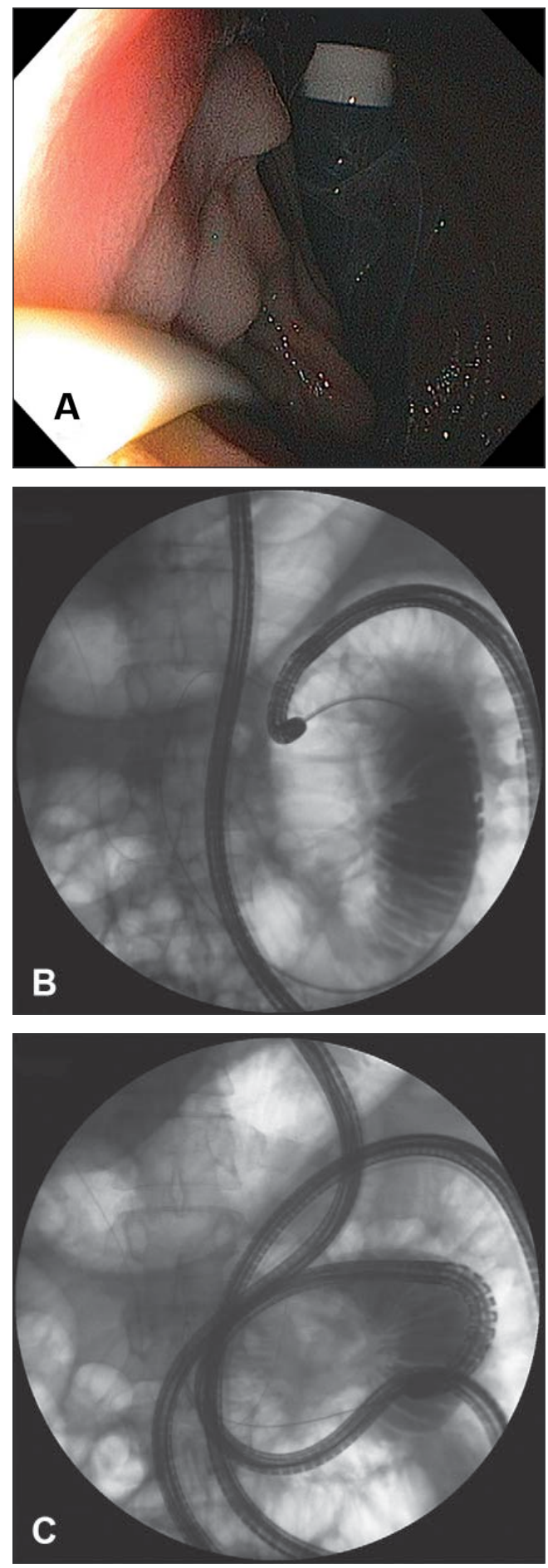

Figura 3. (A) Visión endoscópica del enteroscopio de balón único en el alza yeyunal. (B) Imagen radiológica de la progresión del enteroscopio de balón único sobre hilo guía. (C) Enteroscopio de balón único con múltiples loops objetivando el alza biliar en Y de Roux. Andrew Y. Wang. et al 2010. caciones pertinentes sugieren el empleo de esta técnica, especialmente con el concurso de accesorios adecuados y de especialistas dedicados.

\section{Uso de gastrostomía para acceso a la papila duodenal}

La descripción del uso de la gastrostomía en el estómago excluido después de un BGYR se realizó por primera vez en $1998^{25}$, y desde entonces, hay relatos del uso de ostomías para la realización de CPRE ${ }^{26,27}$.

Matlock et $\mathrm{al}^{28}$, comunican 14 pacientes sometidos a CPRE vía gastrostomía. En 13 pacientes, la CPRE fue realizada en el mismo acto operatorio con un duodenoscopio estéril, inmediatamente después de la exteriorización quirúrgica del estómago; 10 de estas gastrostomías fueron realizadas por vía laparoscópica y se tuvo éxito en la realización de la colangiografía en todos los casos. Martínez et al $^{29}$, describieron la realización de este procedimiento mediante la realización de una gastrostomía percutánea por radiología intervencionista en seis pacientes con BGYR, siendo realizada en tres pacientes la CPRE con duodenoscopio terapéutico, con éxito en la cateterización en dos de ellos.

En 2006, Baron et $\mathrm{al}^{26}$, propusieron el empleo del enteroscopio de doble balón para el posicionamiento retrógrado de la gastrostomía (EPRG) y, a partir de esto, utilizarla para permitir el acceso subsecuentes a CPRE en pacientes con BGYR. Atingiéndose la cámara gástrica exclusa con el enteroscopio, obtiene la transiluminación y la EPRG es realizada de manera semejante a la técnica de la gastrostomía radiológica.

La evaluación de las series de casos publicadas permite inferir que el uso de la gastrostomía es accesible y seguro, permitiendo la realización de duodenoscopia diagnóstica y terapéutica. Una desventaja del método puede ser el tiempo necesario para la maduración de la ostomía, limitando su aplicación en situaciones de urgencia. Por otro lado, existen además todas las desventajas relacionadas a la presencia de ostomía.

\section{Gastrostomía laparoscópica para acceso papila duodenal}

El acceso laparoscópica para la realización de CPRE en paciente con Y- de-Roux ha presentado un incremento en los reportes debido al mayor número de BGYR realizados para el tratamiento de la obesidad. En esta técnica, la interacción entre el cirujano y el endoscopista es fundamental para la realización del procedimiento.

La técnica consiste en identificar el estómago excluido, realizar una gastrostomía e introducir un trocar de 15 a $18 \mathrm{~mm}$ en el cuadrante superior izquierdo. Por este último, se introduce, con el auxilio del cirujano, el duodenoscopio estéril, por la gastrostomía, alcanzándose entonces la papila duodenal mayor ${ }^{11}$.

Ceppa et $\mathrm{al}^{30}$, sometieron 10 pacientes con BGYR a una CPRE transgástrica laparoscópica, identificando 


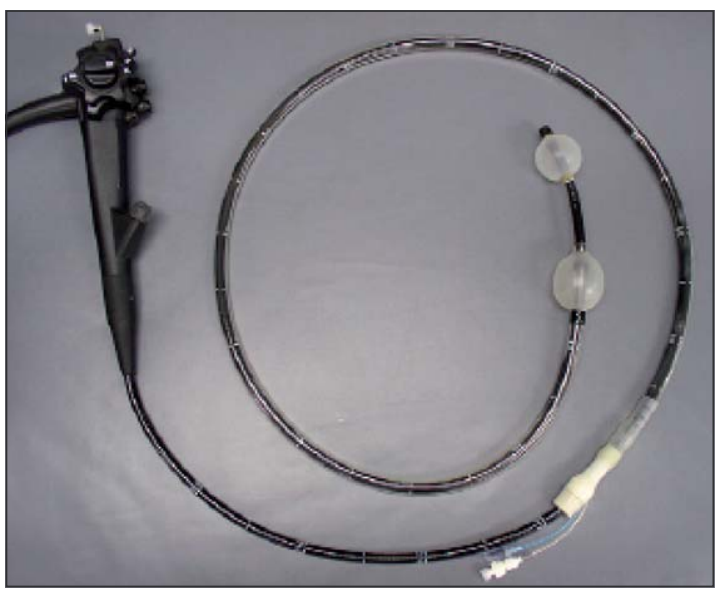

Figura 4. Enteroscopio de doble balón.

enfermedad biliopancreática en cinco de ellos: en cuatro casos (80\%), hubo éxito terapéutico en la canulación y papilotomía. En sólo un paciente no fue posible la canulación debido al impacto del cálculo en la papila. Lopes et $\mathrm{al}^{31}$, presentaron una serie de 10 pacientes sometidos a CPRE asistido por laparoscopia, nueve después de un BGYR y uno después de una antrectomía y reconstrucción con asa en Y-de-Roux. Los accesos endoscópicos fueron vía transgástrica $(\mathrm{n}=9)$ o por la asa biliopancreática $(\mathrm{n}=1)$. Hubo $90 \%$ de éxito en la cateterización y $100 \%$ en la esfinterotomía, ocurriendo pancreatitis moderada en dos pacientes y neumotórax intraoperatorio en un caso, ambos resueltos en el mismo momento. Patel et $\mathrm{al}^{11}$, con ocho pacientes exclusivamente sometidos a BGYR para tratamiento de la obesidad, siendo seis pacientes por laparoscopia y dos por cirugía abierta, realizaron gastrostomía para acceso a la papila duodenal. Obtuvieron éxito terapéutico en todos los casos, sin complicaciones.

Esta estrategia es segura y accesible en la mayoría de los casos. El uso de material endoscópico habitual también hace el procedimiento más factible, además de evaluarse la cavidad abdominal directamente con la posibilidad de tratarse hernias internas o bridas. En las complicaciones relatadas en las series publicadas hay índices aceptables, habiendo riesgo inherente a la anestesia y a la cirugía laparoscópica, no identificando mortalidad. La desventaja es la mayor movilización del equipo quirúrgico y endoscópico, mayor tiempo de ejecución y costo elevado debido al carácter endoscópico-quirúrgico.

\section{Conclusiones}

La evidencia disponible sobre estrategias disponibles para acceder al asa biliopancreática y por tanto, a la papila duodenal mayor en pacientes con una reconstrucción digestiva con un asa en Y-de-Roux es variada y limitada. La mayoría de ellas presentan un bajo porcentaje de morbilidad y es factible en la mayor parte de los pacientes.

Otro punto a resaltar, es que la mayoría de los estudios con los procedimientos relatados fueron realizados en centros de referencia con apoyo de un equipo quirúrgico, reforzando el concepto de que el tratamiento de las enfermedades biliopancreáticas en paciente con anatomía postquirúrgica debe contar con la participación conjunta del cirujano y del endoscopista.

Con el creciente número de operaciones para el tratamiento de la obesidad en todo el mundo, principalmente el BGYR, y la conocida asociación con enfermedad biliopancreática litiásica, la necesidad de una intervención o procedimiento sobre la vía biliar se convertirán en una situación clínica cada vez más común.

El desarrollo de equipamientos y accesorios específicos para efectuar este tipo de procedimientos permitirá aumentar la seguridad y la tasas de éxitos en la realización de estos procedimientos.

\section{Agradecimientos}

Al Dr. Fauze Maluf-Filho por la revisión crítica del artículo.

\section{Referencias}

1. Feitoza AB, Baron TH. Endoscopy and ERCP in the setting of previous upper GI tract surgery. Part I: reconstruction without alteration of pancreaticobiliary anatomy. Gastrointest Endosc. 2001;54:743-9.

2. Taha MI, Freitas WR, Jr., Puglia CR, Lacombe A, Malheiros CA. [Predictive factors for cholelithiasis in the morbidly obese submitted to Roux-en-Y gastroplasty]. Rev Assoc Med Bras. 2006;52:430-4.

3. Pontiroli AE, Morabito A. Long-term prevention of mortality in morbid obesity through bariatric surgery. a systematic review and meta-analysis of trials performed with gastric banding and gastric bypass. Ann Surg. 2011;253:484-7.

4. Veyrie N, Servajean S, Berger N, Loire P, Basdevant A, Bouillot JL. [Gallbladder complications after bariatric surgery]. Gastroenterol Clin Biol. 2007;31:378-84.

5. Desbeaux A, Hec F, Andrieux S, Fayard A, Bresson $\mathrm{R}$, Pruvot $\mathrm{MH}$, et al. Risk of biliary complications in bariatric surgery. J Visc Surg. 2010;147:e217.

6. Laokpessi A, Bouillet P, Sautereau D, Cessot F, Desport JC, Le Sidaner A, et al. Value of magnetic resonance cholangiography in the preoperative diagnosis of common bile duct stones. Am J Gastroenterol. 
2001;96:2354-9.

7. Adler DG, Baron TH, Davila RE, Egan J, Hirota WK, Leighton JA, et al. ASGE guideline: the role of ERCP in diseases of the biliary tract and the pancreas. Gastrointest Endosc. 2005;62:1-8.

8. Shojaiefard A, Esmaeilzadeh M, Ghafouri A, A. M. Various techniques for the surgical treatment of common bile duct stones: a meta review. Gastroenterol Res Pract. 2009;2009:840208. Epub 2009 Aug 6.

9. Chu YC, Yang CC, Yeh YH, Chen $\mathrm{CH}$, Yueh SK. Double-balloon enteroscopy application in biliary tract disease-its therapeutic and diagnostic functions. Gastrointest Endosc. 2008;68:585-91.

10. Emmett DS, DB. M. Double-balloon ERCP in patients who have undergone Roux-en-Y surgery: a case series. Gastrointest Endosc. 2007;66:1038-41.

11. Patel JA, Patel NA, Shinde T, Uchal M, Dhawan MK, Kulkarni A, et al. Endoscopic retrograde cholangiopancreatography after laparoscopic Roux-en-Y gastric bypass: a case series and review of the literature. Am Surg. 2008; 74:689-93; discussion 93-4.

12. Zollinger RM, Jr. The atlas of surgical operations: Elliott Carr Cutler and Robert Zollinger. Am J Surg. 2003;186:211-6.

13. Hintze RE, Adler A, Veltzke W, Abou-Rebyeh H. Endoscopic access to the papilla of Vater for endoscopic retrograde cholangiopancreatography in patients with Billroth II or Roux-en-Y gastrojejunostomy. Endoscopy. 1997;29:69-73.

14. Wright BE, Cass OW, Freeman ML. ERCP in patients with long-limb Roux-en-Y gastrojejunostomy and intact papilla. Gastrointest Endosc. 2002;56:225-32.

15. Gostout CJ, Bender CE. Cholangiopancreatography, sphincterotomy, and common duct stone removal via Roux-en-Y limb enteroscopy. Gastroenterology. 1988;95:156-63.

16. Elton E, Hanson BL, Qaseem T, DA. H. Diagnostic and therapeutic ERCP using an enteroscope and a pediatric colonoscope in long-limb surgical bypass patients. Gastrointest Endosc. 1998;47:62-7.

17. Neumann H, Fry LC, Meyer F, Malfertheiner P, K. M. Endoscopic retrograde cholangiopancreatography using the single balloon enteroscope technique in patients with Roux-en-Y anastomosis. Digestion. 2009;80:52-7.

18. Wang AY, Sauer BG, Behm BW, Ramanath M, Cox DG, Ellen KL, et al. Single-balloon enteroscopy effectively enables diagnostic and therapeutic retrograde cholangiography in patients with surgically altered anatomy. Gastrointest Endosc. 2010;71:641-9.
19. Saleem A, Baron TH, Gostout CJ, Topazian MD, Levy MJ, Petersen BT, et al. Endoscopic retrograde cholangiopancreatography using a single-balloon enteroscope in patients with altered Roux-en-Y anatomy. Endoscopy. 2010;42: 656-60.

20. Hartmann D, Eickhoff A, Tamm R, JF. R. Balloonassisted enteroscopy using a single-balloon technique. Endoscopy. 2007;39(Suppl 1):E276.

21. Parlak E, Cicek B, Disibeyaz S, Cengiz C, Yurdakul M, Akdogan M, et al. Endoscopic retrograde cholangiography by double balloon enteroscopy in patients with Roux-en-Y hepaticojejunostomy. Surg Endosc. 2010;24:466-70.

22. Aabakken L, Bretthauer M, Line PD. Double-balloon enteroscopy for endoscopic retrograde cholangiography in patients with a Roux-en-Y anastomosis. Endoscopy. 2007;39:1068-71.

23. Kuga R, Furuya CK Jr, Hondo FY, Ide E I, Shioka S, P. S. ERCP using double-balloon enteroscopy in patients with Roux-en-Y anatomy. Dig Dis. 2008;26:330-5.

24. Monkemuller K, Fry LC, Bellutti M, Neumann H, Malfertheiner P. ERCP with the double balloon enteroscope in patients with Roux-en-Y anastomosis. Surg Endosc. 2009;23:1961-7.

25. Baron TH, Vickers SM. Surgical gastrostomy placement as access for diagnostic and therapeutic ERCP. Gastrointest Endosc. 1998;48:640-1.

26. Baron TH. Double-balloon enteroscopy to facilitate retrograde PEG placement as access for therapeutic ERCP in patients with long-limb gastric bypass. Gastrointest Endosc. 2006;64:973-4.

27. Ross AS, Dye C. Double-balloon enteroscopy to facilitate retrograde PEG placement as access for therapeutic ERCP in patients with long-limb gastric bypass. Gastrointest Endosc. 2007;66:419; author reply 20.

28. Matlock J, Ikramuddin S, Lederer H, O. C. Bypassing the Bypass: ERCP Via Gastrostomy after Bariatric Surgery. Gastrointest Endosc. 2005;61:AB98.

29. Martínez J, Guerrero L, Byers P, López P, Scagnelli T, Azuaje R, et al. Endoscopic retrograde cholangiopancreatography and gastroduodenoscopy after Roux-en-Y gastric bypass. Surg Endosc. 2006;20:1548-50.

30. Ceppa FA, Gagne DJ, Papasavas PK, Caushaj PF. Laparoscopic transgastric endoscopy after Roux-en-Y gastric bypass. Surg Obes Relat Dis. 2007;3:21-4.

31. Lopes TL, Clements RH, Wilcox CM. Laparoscopyassisted ERCP: experience of a high-volume bariatric surgery center (with video). Gastrointest Endosc. 2009;70:1254-9. 